\title{
Uso inadecuado de opioides en pacientes con dolor oncológico: revisión sistemática integradora de la literatura
} \author{
Opioid misuse in patients with cancer pain: an integrative \\ systematic review of the literature
}

\author{
M. Labori Trias ${ }^{1}$, R. Hernández-Ribas² y J. Porta-Sales ${ }^{1,3}$
}

'Servicio de Cuidados Paliativos, Institut Català d'Oncologia. Hospitalet de Llobregat, Barcelona, España. ¿Servicio de Psiquiatría, Hospital Universitari Bellvitge. Institut Català d'Oncologia. CIBERSAM. Universitat de Barcelona, España. ${ }^{3}$ Universitat Internacional de Catalunya. Barcelona, España

\section{ABSTRACT}

Background: Opioids are widely used for the treatment of cancer pain and non-malignant pain. There is a lot of information about opioid misuse (OM) in patients with non-malignant pain, however in cancer patients there is less evidence.

Objectives: To identify, appraise and synthesize existing evidence about epidemiology, risk factors, clinical tools and evolution of $\mathrm{OM}$ in patients with cancer pain.

Methods: Integrative systematic review with data extraction and narrative synthesis. PubMed, Web of Science y PsychINFO databases were searched for articles published through 31 December 2017. Study inclusion criteria were as follows: 1) published in English, Spanish or French language; and 2) containing data on the prevalence or incidence of $\mathrm{OM}$ in patients with cancer pain; or/and 3) providing information about OM risk factors, mortality, duration and remission.

Results: The search yielded 3520 articles, of which 40 met the inclusion criteria. Four themes were identified: 1) epidemiology, 2) risk factors, 3) patient's and professional's opinion, and 4) specific policies. The obtained results were very heterogeneous; the prevalence of $\mathrm{OM}$ varied from $\mathrm{O}$ up to $26 \%$ and the prevalence of opioid-associated aberrant behaviours from 12 to $85 \%$. Different risk factors for OM were identified, highlighting young age and history of psychiatric disorders or substance abuse, and different tools for

Labori Trias M, Hernández-Ribas R, Porta-Sales J. Uso inadecuado de opioides en pacientes con dolor oncológico: revisión sistemática integradora de la literatura. Rev Soc Esp Dolor. 2020;27(5):306-315

\section{RESUMEN}

Introducción: Los opioides son ampliamente utilizados para el control del dolor oncológico y no oncológico. Existe mucha información sobre el uso inadecuado de opioides (UIO) en pacientes no oncológicos, sin embargo en pacientes oncológicos existe menos evidencia.

Objetivos: Identificar, analizar y sintetizar la evidencia disponible sobre la epidemiología, los factores de riesgo, instrumentos clínicos y evolución del UIO en pacientes con dolor oncológico.

Material y métodos: Revisión sistemática integradora de la literatura con extracción de datos y síntesis narrativa. Las fuentes utilizadas para buscar artículos publicados hasta el 31 de diciembre de 2017 fueron las bases de datos PubMed, Web of Science y PsychINFO. Se eligieron aquellos artículos que siguieran los siguientes criterios de inclusión: 1] publicados en lengua inglesa, española o francesa, y 2) que incluyesen información sobre la prevalencia o incidencia del UIO en pacientes con dolor oncológico, o/y 3) que aportasen información sobre factores de riesgo, mortalidad, duración del UIO y su remisión.

Resultados: De la búsqueda surgieron 3520 artículos, de los cuales 40 cumplieron los criterios de inclusión. Se identificaron cuatro áreas temáticas: 1) epidemiología, 2) factores de riesgo, 3) opinión de pacientes y profesionales, y 4) políticas específicas. Los resultados obtenidos fueron muy heterogéneos, iden-

Recibido: 12-03-2020

Aceptado: 06-06-2020

Correspondencia: Maria Labori Trias mlabori@iconcologia.net 
risk assessment or diagnosis of OM were described. Regarding professional's opinion, it seems to be more awareness about $\mathrm{OM}$ but nevertheless policies regarding this problem are scarce.

Conclusions: Even though there is a greater awareness among professionals about $\mathrm{OM}$ in cancer patients, the current information is very heterogeneous and does not allow clear conclusions. For this reason, it will be necessary to carry out new studies trying to standardize criteria and establish better protocols and policies for detection and management of $\mathrm{OM}$.

Keywords: Cancer pain, analgesics opioids, opioid-related disorders, epidemiology, risk factors. tificándose una prevalencia que oscila entre el $\mathrm{O}$ y el $26 \%$ en el UIO y entre el 12 y el $85 \%$ en las conductas aberrantes relacionadas con opioides. Se identificaron diferentes factores de riesgo de UIO, destacando la edad joven y la presencia de antecedentes psiquiátricos o de abuso de sustancias, y se describieron diferentes instrumentos dirigidos a la evaluación del riesgo o bien al diagnóstico del UIO. En cuanto a la opinión de los profesionales, parece haber un incremento de la sensibilización al respecto, pero las políticas de los centros en relación con esta problemática suelen ser casi inexistentes.

Conclusiones: A pesar de que existe una mayor sensibilización de los profesionales acerca del UIO en pacientes oncológicos, la información de que disponemos es muy heterogénea y no nos permite extraer conclusiones claras. Por esta razón será necesaria la realización de nuevos estudios intentando homogeneizar criterios y establecer mejores protocolos y políticas de detección e intervención frente al UIO.

Palabras clave: Dolor oncológico, opioides, uso inadecuado, epidemiología, factores de riesgo.

\section{INTRODUCCIÓN}

Es ampliamente conocido que los opioides son sustancias capaces de producir trastornos adictivos, al igual que sucede con otras drogas como el alcohol, las benzodiacepinas o la cocaína. La última edición del Manual Diagnóstico y Estadístico de los Trastornos Mentales (DSM-5) describe al "trastorno por consumo de opioides" como el conjunto de signos y síntomas que reflejan la autoadministración prolongada y compulsiva de opioides que no se utilizan para un propósito médico legítimo 0 , si existe otra afección médica que requiere tratamiento con opioides, por la utilización en dosis muy superiores a la cantidad necesaria para dicha afección médica [1]. Si nos centramos solo en los trastornos relacionados con los opioides prescritos médicamente, y de forma independiente del DSM-5, en la literatura han hecho fortuna los conceptos "uso inadecuado de opioides", que se podría definir como el uso de dicha medicación de forma distinta a la pautada en el plan terapéutico, y "comportamiento aberrante relacionado con opioides", que incluiría cualquier conducta que reflejase un uso inadecuado $[2,3]$. La presencia o ausencia de conductas aberrantes en el consumo de opioides prescritos es un punto fundamental a la hora de valorar la respuesta clínica a dicho tratamiento y, de hecho, forma parte de las llamadas 4A's que definen el éxito del tratamiento con opioides: 1) la Analgesia obtenida; 2) la posibilidad de realizar las Actividades de la vida diaria; 3) la ausencia de efectos Adversos, y 4) la ausencia de comportamientos "Aberrantes" en la toma de opioides (4). Las conductas "aberrantes" incluirían en sí mismas diferentes situaciones, definidas en inglés como: "misuse" (mal uso), "abuse” (abuso), "addiction” (adicción), "diversion" (desvío) y "chemical copying" (afrontamiento químico) $[2,3,5)$ (ver definiciones en Tabla I). Merece especial atención la conducta de afrontamiento químico, que puede o no formar parte de una adicción y que se define como el consumo por encima de los límites aconsejados con finalidad ansiolítica y acompañado de conductas compulsivas y destructivas. En el caso de los pacientes con cáncer dicha conducta de afrontamiento químico podría tener como objetivo manejar la ansiedad que implica la enfermedad y el dolor [5].

Uno de los aspectos centrales en el uso inadecuado de opioides es la valoración clínica del mismo y, en este sentido, se han desarrollado diversas herramientas de evaluación. Los instrumentos existentes hasta la fecha se podrían dividir en dos grandes grupos: los dirigidos a la estimación del riesgo de incumplir la prescripción médica de opioides, siendo los más utilizados el "CAGEAID", modificación del cuestionario CAGE encaminado a la detección del consumo problemático de drogas como los opioides [6], el "Opioid Risk Tool" (ORT) [7], y el "Screener and Opioid Assessment for Patients with Pain" (SOAPP) (8), y los centrados en la detección y monitorización del uso inadecuado ya establecido, donde destacaría el "Current Opioid Misuse Measure" [9]. Es importante puntualizar que, a fecha de hoy, ninguna de estas herramientas está validada al español.

En cuanto a la prevalencia del uso inadecuado de opioides, cabe resaltar que en la última década diversos autores (10-12) y entidades, como el Departamento de Justicia de los Estados Unidos (13), han llamado la atención sobre el incremento, cercano al $150 \%$, en el consumo de opioides y su uso inadecuado, especialmente en pacientes con dolor crónico no oncológico en Estados Unidos (EE. UU.), hecho que condiciona, 
TABLA I

DIFERENTES SITUACIONES DE USO DE OPIOIDES INCLUIDAS EN EL CONCEPTO DE CONDUCTA "ABERRANTE" (3)

\begin{tabular}{|l|l|l|}
\hline \multicolumn{1}{|c|}{ Término inglés } & \multicolumn{1}{|c|}{ Término español } & \multicolumn{1}{c|}{ Definición } \\
\hline Misuse & Mal uso & $\begin{array}{l}\text { Uso de un opioide con intención terapéutica diferente a la } \\
\text { indicada, ya sea intencionada o no, con o sin efectos adversos } \\
\text { para el paciente }\end{array}$ \\
\hline Abuse & Abuso & $\begin{array}{l}\text { Uso intencionado de un opioide prescrito o no prescrito para } \\
\text { cualquier finalidad no médica }\end{array}$ \\
\hline Addiction & $\begin{array}{l}\text { Es una enfermedad neurobiológica crónica cuyas } \\
\text { manifestaciones y evolución se ven influenciadas por factores } \\
\text { genéticos, psicosociales y ambientales. Se caracteriza por: } \\
\text { deficiente control sobre el uso, uso compulsivo, uso continuado } \\
\text { a pesar del daño, y ansiedad por obtener el fármaco (craving) }\end{array}$ \\
\hline Diversion & Desvío & $\begin{array}{l}\text { Desvío intencionado de opioide/s de los canales legales de } \\
\text { dispensación y control }\end{array}$ \\
\hline Polerance & $\begin{array}{l}\text { Estado que se manifiesta por un síndrome de abstinencia que } \\
\text { aparece ante la retirada abrupta del opioide, una reducción } \\
\text { rápida de su dosis, el descenso de sus niveles plasmáticos o la } \\
\text { administración de un antagonista }\end{array}$ \\
\hline Tolerancia & $\begin{array}{l}\text { Estado en que hay una disminución del efecto terapéutico del } \\
\text { opioide, tras un periodo de uso prolongado con la necesidad } \\
\text { de incrementar sus dosis para obtener el mismo efecto } \\
\text { farmacológico previo }\end{array}$ \\
\hline
\end{tabular}

además, un incremento global de los costos sanitarios asociados sin evidencia de beneficio clínico, e incremento de las comorbilidades relacionadas a los opioides. En EE. UU. se ha estimado un incremento de hasta un $43 \%$ en el uso inadecuado de opioides en la primera década de este siglo, con un incremento paralelo de muertes no intencionales por opioides (14). En Canadá, se describen situaciones parecidas a las de los EE. UU., reportándose que de entre todos los fallecimientos relacionados con el uso de fármacos, entre 2006 y 2008 en Ontario, el 58 \% tenían relación con el uso de opioides, mayoritariamente (93\%) en pacientes no oncológicos (15). Progresivamente, esta alarma se ha ido extendiendo a Europa (16), y en los últimos años los estudios se han empezado a ocupar de los pacientes con dolor oncológico [17].

En España, respondiendo a esta inquietud general, se realizó en 2017 la "Guía de consenso para el buen uso de analgésicos opioides", resultado del trabajo de cuatro sociedades científicas: Socidrogalcohol, la Sociedad Española de Medicina de Familia y Comunitaria (SEMFyC), la Federación de Asociaciones de Enfermería Comunitaria y Atención Primaria (FAECAP) y la Sociedad Española de Cuidados Paliativos (SECPAL) (18). Dicha guía intenta dar pautas de actuación estándar, tanto a nivel de prevención como de abordaje global, para el uso inadecuado de opioides prescritos médicamente.

En cualquier caso, y a pesar de la razonable alarma suscitada, es importante puntualizar que la gran mayoría de información disponible hace referencia al uso de opioides en pacientes con dolor crónico de origen no oncológico. De hecho, los estudios que analizan el uso inadecuado de opioides prescritos en pacientes con cáncer lo describen como muy poco frecuente, ya sea en el manejo del dolor basal como del irruptivo (19-21). Las dos primeras revisiones realizadas en pacientes con cáncer determinaron que la información que se disponía era poco concluyente, oscilando la prevalencia de adicción a opioides entre el $\mathrm{O}$ y el 7,7 \%, en función de los estudios analizados $[22,23]$. De todas formas, una revisión más reciente concluye que uno de cada cinco pacientes oncológicos estaría en riesgo de presentar un uso inadecuado de opioides prescritos, cifra de franca relevancia clínica que aconseja profundizar en el estudio de este proceso (17).

Por todo lo anterior, consideramos que es preciso aportar mayor evidencia sobre el potencial uso inadecuado de opioides en pacientes con cáncer. Así pues, el objetivo de la presente revisión es revisar y sintetizar de forma sistemática la evidencia disponible sobre la frecuencia, tipo y factores de riesgo asociados con el uso inapropiado de opioides en pacientes con dolor oncológico.

\section{MATERIAL Y MÉTODOS}

\section{Diseño y fuente de los datos}

Se ha realizado una revisión sistemática siguiendo la guía PRISMA (Preferred Reporting Items for Systematic Reviews and Meta-Analysis] [24]. La búsqueda de la literatura se hizo en las siguientes bases de datos: PubMed, Web of Science y PsychINFO, evaluándose 
todas las publicaciones desde su puesta en marcha hasta el 31 de diciembre de 2017.

\section{Criterios de elegibilidad}

Se consideraron como elegibles aquellos artículos en lengua inglesa, española o francesa que incluyesen información sobre la prevalencia o incidencia del uso inadecuado de opioides en pacientes con dolor oncológico y/o que aportasen información sobre factores de riesgo, mortalidad, duración del uso inadecuado y su remisión. No se incluyeron editoriales ni cartas al director, ni series de casos, ni revisiones no sistemáticas.

\section{Estrategia de búsqueda y selección de estudios}

La estrategia de búsqueda para la base de datos PubMed, que utiliza tanto términos MeSH como palabras clave que no eran términos $\mathrm{MeSH}$, se muestra en el Anexo 1. Siguiendo el mismo modelo, se adaptó la estrategia de búsqueda para cada base de datos. Los artículos identificados siguieron un proceso de selección realizado por tres evaluadores (M. L., R. H-R. y
J. P-S.) en tres fases: inicialmente por título, posteriormente por abstract y finalmente por texto completo. Además, se realizó una búsqueda manual de las referencias de los artículos identificados. Se excluyeron aquellos artículos que no cumplían los criterios de inclusión mencionados previamente y, en caso de duda, se resolvió mediante acuerdo entre los tres revisores. La Figura 1 muestra el proceso de búsqueda y selección de los artículos.

\section{Extracción y análisis de los datos y síntesis de los resultados}

Los datos se extrajeron y sintetizaron utilizando el método integrador descrito por Whittemore y Knafl (25), que permite la síntesis de diversas fuentes incluyendo tanto trabajos empíricos (cualitativos, cuantitativos y métodos mixtos] como estudios teóricos (casos, modelos teóricos, comentarios y reflexiones]. El proceso de análisis de los datos mediante esta metodología permite integrar los hallazgos de todos los estudios incluidos. Todos los estudios que cumplieron los criterios de inclusión se trataron de la misma forma en esta síntesis. Se utilizó, también, el proceso de análisis de datos propuesto por Whittemore y Knafl (25), que inclu-

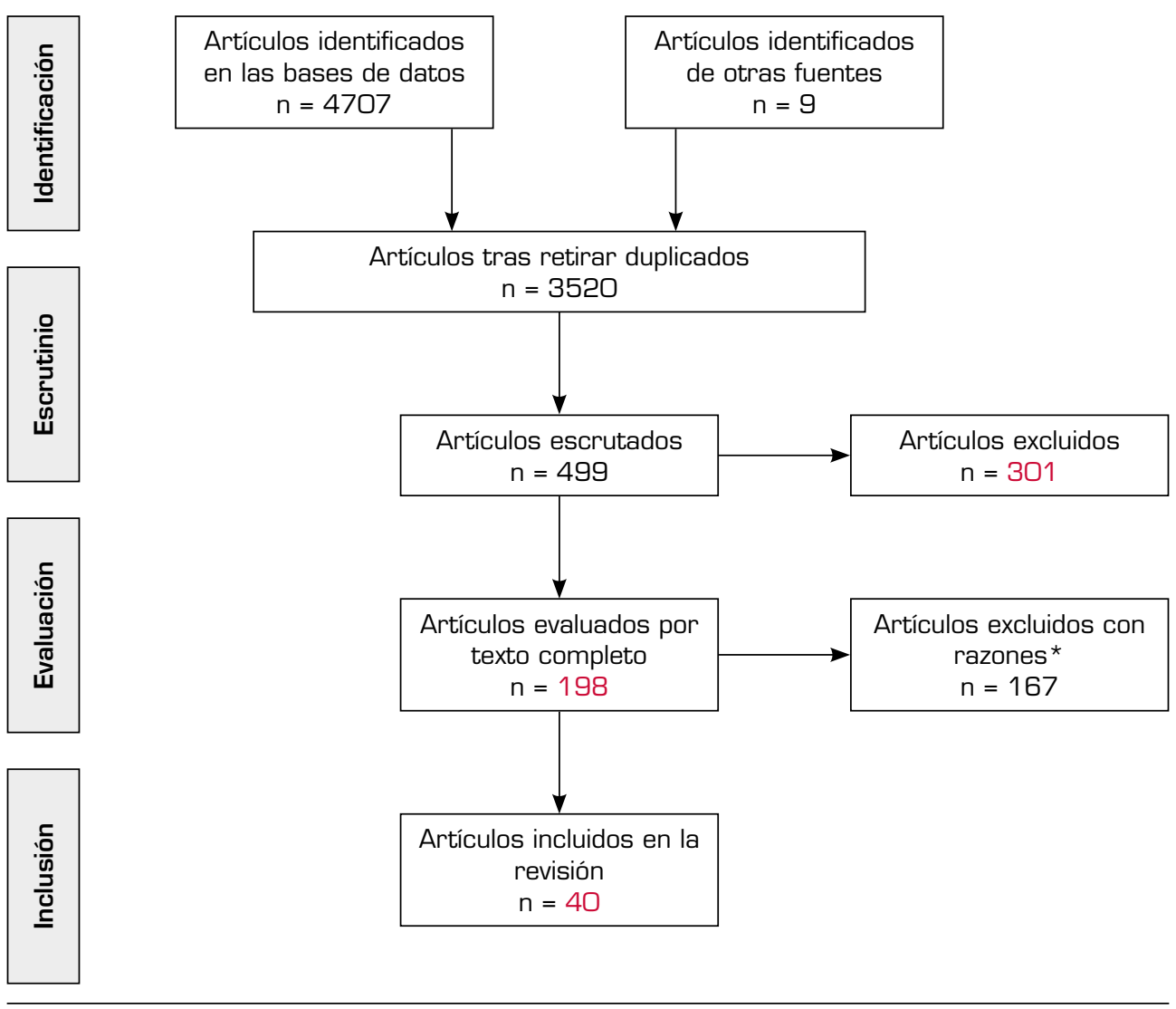

Fig. 1. Diagrama de flujo PRISMA de la selección de estudios.

* Razones para exclusión: a) Idioma no inglés ni francés ( $n=2$ ); b) No cumplían criterios inclusión ( $n=126)$; c) No incluía pacientes oncológicos ( $n=35)$; d) Serie de 2 casos $(n=4)$. 
ye la síntesis, la presentación y la comparación de los datos, así como llegar a conclusiones. El primer paso es desarrollar una matriz para extraer las principales características de cada uno de los artículos incluidos: autor/es, año de publicación, país donde se realizó el estudio, tipo de estudio, número de pacientes incluidos, objetivo principal, opioide/s evaluado/s, y resultados principales. Tras el análisis de la matriz de datos emergieron cuatro temas relacionados con los objetivos de la presente revisión: 1] prevalencia/incidencia, 2) factores de riesgo e instrumentos para la evaluación, 3) opinión de los profesionales y de los pacientes, y 4) políticas específicas sobre el uso inadecuado de opioides llevadas a cabo. A continuación, todos los artículos que cumplieron los criterios de inclusión se clasificaron dentro de alguna de las cuatro categorías anteriores y fueron analizados. Los datos de cada artículo se extrajeron y se compilaron en la matriz de datos, independientemente del grado de evidencia que aportaban. El tercer paso fue la comparación punto por punto de los datos extraídos con el fin de garantizar que se comparaban, categorizaban y sintetizaban datos similares. Los datos se manejaron manualmente para organizar y facilitar el análisis.

\section{Análisis de la calidad}

Se siguió la guía PRISMA para comunicar las características de los estudios y su calidad metodológica (24). Debido a la falta de consenso en la literatura respecto a la exclusión de estudios por razones metodológicas [26], se decidió no excluir ningún estudio por cuestiones de calidad.

\section{RESULTADOS}

De los 3520 artículos identificados inicialmente se revisaron por texto completo un total de 198, de los cuales se excluyeron 167 (ver las razones de exclusión en la Figura 1). Cuarenta de las publicaciones cumplieron los criterios de inclusión y fueron incluidas en la revisión. En el Anexo 2 se muestran las principales características de los 40 artículos escogidos con un resumen de los principales hallazgos. Las metodologías de los artículos incluidos en la revisión son diversas: revisiones sistemáticas ( $n=2 ; 5 \%$ ), estudios retrospectivos ( $n=12 ; 30 \%$ ), prospectivos ( $n=8 ; 20 \%$ ), transversales ( $n=15,37,5 \%$ ), estudios cualitativos ( $n=2 ; 5 \%$ ) y estudios Delphi $(n=1 ; 2,5 \%$ ). Veintidós (55\%) artículos se han publicado en EE. UU., 9 [22,5\%) en países europeos, 3 (7,5 \%) en países asiáticos, 1 [2,5\%) en Egipto, 1 [2,5\%) en Australia, 1 (2,5\%) en Brasil y 3 (7,5\%) son trabajos internacionales. En cuanto al tipo de opioide analizado en los diferentes trabajos cabe resaltar que en 25 (62,5\%) se incluye a todos los opioides sin especificar ninguno en concreto, que en $7(17,5 \%)$ de los estudios se analiza la dosis equivalente de morfina diaria, en 3 [7,5 \%) se describe el tratamiento de cada paciente pero en los resultados no se diferencia entre los distintos opioides y solo 5 (12,5\%) de los estudios se centran en un solo opioide (ver detalle en Anexo ?).
A continuación se procederá a describir los resultados de los artículos revisados clasificados en cada una de las cuatro áreas temáticas emergentes del análisis de la matriz: epidemiología, factores de riesgo, opinión y políticas específicas.

\section{Epidemiología: prevalencia/incidencia}

Los primeros estudios realizados en pacientes oncológicos detectaron una frecuencia muy baja de adicción a analgésicos opioides, describiéndose cifras de incidencia del $0 \%$ en seguimientos de 3 y 6 meses [27), y de prevalencia inferiores al $5 \%$ tanto en pacientes adultos como pediátricos $[28,29]$. En la misma línea, un trabajo que recogía la experiencia de 100 médicos de atención primaria concluía que únicamente un $1 \%$ de estos detectaba pacientes con dependencia a opioides, con cifras de incidencia que oscilaban entre 10 2 pacientes al año [30). Un estudio más reciente, en cambio, ha reportado una prevalencia mayor de dependencia a opioides, situándola en el 5,65 \%, aunque debemos puntualizar que en este caso no se especificaba si los opioides eran prescritos médicamente o bien se trataba de opioides ilegales (31).

En los últimos años se ha acuñado el término "Uso inadecuado de opioides", que tal como referíamos en la introducción, incluye diversos conceptos a parte de la adicción como son el mal uso, el abuso, el desvío o el afrontamiento químico. Entre los estudios realizados bajo este paraguas cabría destacar un trabajo prospectivo que concluye que el $90 \%$ de los pacientes oncológicos cumplen la prescripción pautada de opioides [20], un estudio retrospectivo que detecta tan solo un $0,1 \%$ de uso inadecuado de opioides en pacientes con cáncer [32] y dos estudios multicéntricos realizados con el objetivo de valorar la seguridad de las presentaciones de fentanilo de absorción transmucosa que no reportan ningún caso de uso inadecuado $(33,34)$.

De forma contrapuesta a estos resultados, algunos estudios reportan incidencias mayores de uso inadecuado y profundizan en las llamadas conductas aberrantes. En este sentido, cabría destacar el trabajo prospectivo realizado por Kwon y cols. [35], que detecta un $18 \%$ de afrontamiento químico en el consumo de opioides prescritos en pacientes con cáncer. Si profundizamos en este dato, es importante referir que la cifra de $18 \%$ es el resultado de la valoración del paciente por un especialista en cuidados paliativos, mientras que al contrastar con la historia clínica general de los mismos pacientes, el porcentaje detectado es tan solo del $4 \%$. Este trabajo nos da información interesante acerca del posible infradiagnóstico del uso inadecuado de opioides en pacientes con cáncer cuando no son evaluados por un profesional con conocimiento amplio del tema. Respecto a las conductas aberrantes que reflejan un uso inadecuado, dos trabajos estudian el tipo de almacenamiento de opioides en domicilio, así como el estilo de uso de los mismos. En dichas publicaciones se reportan unos resultados francamente alarmantes, ya que determinan que más del $85 \%$ de los pacientes oncológicos almacenan de forma no segura los opioides y que entre el 13 y el $26 \%$ de los pacientes realizan un uso inseguro, definido como el uso compartido o la 
pérdida de medicación $[36,37]$. Respecto a pacientes adolescentes y adultos jóvenes con cáncer, dos estudios sitúan la incidencia de uso inadecuado de opioides alrededor de un $12 \%$, siendo las conductas aberrantes más frecuentes la preocupación de una tercera persona por el abuso opioide o por el manejo de los fármacos del paciente y la manera que tiene el paciente de nombrar o hablar del fármaco: si utiliza el nombre comercial o bien alguno procedente del argot callejero $[38,39]$.

Si nos centramos en las revisiones sistemáticas, tan solo dos de ellas incluyen información sobre la epidemiología del uso inadecuado de opioides en pacientes oncológicos: la primera se focaliza en la adicción a opioides en pacientes con dolor crónico (22) y la segunda en el desarrollo de dependencia a opioides como analgésicos [23]. Hojsted y Sjogren [22] consideran 5 estudios realizados en población oncológica (40-44) y describen una frecuencia de "adicción" que oscila entre el 0 y 7,7 \%. Por otra parte, la segunda de las revisiones sistemáticas [23] incluye dos estudios que contemplan a los pacientes con cáncer $(45,46)$ pero no ofrece información concluyente, sobre todo por dificultades metodológicas. En estos dos estudios considerados por Minozzi, es interesante comentar la comparativa realizada por Passik y cols. (45), que muestra una baja frecuencia de "uso inadecuado" de opioides en pacientes con cáncer, cuando se compara con los pacientes con SIDA, donde es 6 veces superior.

\section{Factores de riesgo e instrumentos de evaluación}

Un total de dieciséis estudios [28,35-39,47-56] aportan información respecto a los factores de riesgo de uso inadecuado de opioides. Se han identificado factores relacionados con la edad, teniendo más riesgo aquellos pacientes menores de 55 años $[35,49,50]$. También se han descrito como factores de riesgo la depresión $(39,49,51,53,56)$, los antecedentes personales o familiares de abuso de alcohol $[50,51,53]$, el consumo de sustancias ilegales $[50,56]$ y el dolor intenso $(35,49,56]$. Además, se han descrito factores de riesgo relacionados con los propios opioides entre los que destacarían la presencia de clínica abstinencial intensa al inicio del tratamiento (28), el uso únicamente de medicación de rescate (47) y la prescripción de altas dosis $(47,49)$. En relación a este último punto, se ha visto que el riesgo de uso inadecuado de opioides aumenta cuanto mayor es la dosis de opioide prescrita; si bien no se define una dosis concreta a partir de la cual este riesgo es más elevado, los estudios parecen apuntar que a partir de una dosis equivalente de morfina oral diaria de $50 \mathrm{mg}$ el riesgo es mayor. Finalmente, Silvestre y cols. describen otros factores de riesgo como la situación laboral, la percepción de prescripción excesiva por parte del paciente y el desconocimiento sobre programas de devolución de fármacos (37).

Tal como se refería en la introducción, para la identificación de factores de riesgo vinculados al uso inadecuado de opioides se han utilizado diversos instrumentos como el CAGE, el SOAPP o el ORT. Además, se han realizado trabajos utilizando técnicas de detección precoz como la detección de opioides en orina. A continuación detallaremos los principales datos al respecto.
Varios estudios evalúan el riesgo de uso inadecuado de opioides en pacientes con cáncer relacionándolo con los cuestionarios CAGE y CAGE-AID $[35,36,52-55)$. De los resultados de los estudios analizados, podemos resumir que los pacientes oncológicos fumadores presentan un porcentaje mayor de positivos en el cuestionario CAGE que los no fumadores (55) y que un resultado positivo en dicho instrumento en pacientes con cáncer ha mostrado ser un potente predictor de afrontamiento químico (35), de uso inseguro de opioides [36] y además aumenta la probabilidad de que el profesional sanitario solicite un test de orina (54). Es importante destacar que, curiosamente y de forma aparentemente contraintuitiva, el CAGE positivo es predictor de almacenamiento seguro de opioides [36].

Dos trabajos realizan una evaluación del riesgo mediante el ORT $[50,51]$ y cinco estudios evalúan el riesgo mediante el uso del test SOAPP, dos de ellos utilizan la versión original revisada: SOAPP-R $[38,56]$ y tres de ellos la versión corta: SOAPP-SF $[48,49,52]$. A resaltar que dos de estas publicaciones añaden a las herramientas de estimación de riesgo un test de detección de opioides en orina $[50,52)$. Además, un estudio evalúa el riesgo en 94 pacientes adolescentes y adultos jóvenes mediante una herramienta propia llamada "Screen for Opioid-Associated Aberrant Behavior Risk [SOABR] [39]. Finalmente, en un estudio retrospectivo se analizan los motivos del profesional para solicitar un test de opioides en orina [54], siendo estos: edad menor de 45 años, raza afroamericana, nivel educacional bajo, estadio precoz de la enfermedad, CAGEAID positivo, puntuación alta del dolor, baja puntuación de astenia. Los estudios realizados con el test ORT determinan un riesgo moderado-alto de desarrollar un uso inadecuado de opioides en un $43 \%$ de los pacientes oncológicos $(50,51)$, mientras que un trabajo más reciente que utiliza el SOAPP-R lo sitúa en el $32 \%$ [56]. En cambio, un estudio realizado mediante SOAPP-SF en una población que incluye un $91 \%$ de pacientes oncológicos determina en cambio un riesgo más bajo, entorno el $8 \%$ de los casos [52). De forma francamente interesante, los pacientes clasificados como de riesgo moderado-alto mediante el ORT muestran una alta probabilidad de presentar un test de opioides en orina alterado, describiéndose en el 62,5\% de los pacientes estudiados, hecho que le otorga un potente papel como predictor [50]. Al igual que se ha descrito con el CAGE, los fumadores presentan un mayor riesgo de uso indebido de opioides estimado mediante el SOAPP-SF y además presentan mayores cambios de humor, mayor probabilidad de tomar medicamentos distintos a los recetados, de presentar antecedentes de problemas legales o consumo de drogas ilegales (48). Respecto a los pacientes oncológicos adolescentes y adultos jóvenes, el estudio que evalúa el riesgo mediante el SOAPP-R detecta un 39,5 \% de los pacientes con un riesgo alto y es importante puntualizar que de este grupo, el $33 \%$ desarrolló conductas aberrantes en el seguimiento (38). En el estudio que utiliza el instrumento SOABR, se detectan factores de riesgo para un uso inadecuado en un $90,9 \%$ de los casos, siendo los más frecuentes los antecedentes personales y familiares de trastorno mental y el uso simultáneo de más de un opioide [39]. 


\section{Opinión de los profesionales y de los pacientes}

En la revisión efectuada se han encontrado catorce estudios sobre la opinión de profesionales y pacientes acerca de los opioides o de algún aspecto relacionado con el uso inadecuado de los mismos: cuatro versan acerca de la opinión de profesionales $[30,53,57,58)$, ocho se centran en pacientes con cáncer [29,59-65) y dos en personas de la población general $[66,67]$.

Respecto a la opinión de los profesionales, un estudio de Vainio y cols. concluyó en la década de los ochenta que un $50 \%$ de los médicos de atención primaria referían no detectar nunca pacientes con dependencia a opioides y un $23 \%$ no solían prescribir opioides (30). En la misma línea, se describió que entre los profesionales de hospitales oncológicos, un $61 \%$ de los médicos manifestaban reticencia para prescribir opioides y un $38 \%$ de las enfermeras para administrarlos (57). Estudios posteriores muestran un mayor conocimiento del tema por parte de los profesionales, destacando que los médicos de atención primaria sitúan la prevalencia de dependencia a opioides en pacientes con cáncer entorno al $10 \%$ [58] y que especialistas en Cuidados Paliativos y Dolor muestran un consenso del $92 \%$ en la definición y detección de algunos aspectos concretos del uso inadecuado de opioides como el afrontamiento químico frente al malestar emocional [53].

En relación con lo que piensan al respecto los propios pacientes oncológicos, un $52 \%$ considera que fácilmente pueden presentar adicción a opioides [60] y un 55,6 \% manifiesta preocupación por presentar adicción [59), siendo esta preocupación una de las barreras más frecuentes en el manejo del dolor [63]. Algunos pacientes relacionan el uso de la morfina con el final de vida, hecho que también puede dificultar el manejo del dolor mediante opioides [62). Respecto al dolor irruptivo, un $81 \%$ de los pacientes preguntados en un trabajo de Davies y cols. refieren dificultad para la toma de opioide de rescate por preocupaciones acerca de la posibilidad de presentar tolerancia, sobredosis o adicción [61]. Además, puede existir alguna diferencia transcultural en la percepción de los pacientes, ya que los estudios realizados en diferentes países nos aportan resultados distintos. Así, en un trabajo realizado en Egipto se concluye que un 50,6 \% de los pacientes rechazarían tramadol por miedo a la adicción (64) y otro centrado en Brasil detecta un 19,2\% de pacientes que rechazarían un tratamiento con morfina por miedo a desarrollar una dependencia [65); sin embargo, en un estudio en pacientes pediátricos suecos, ningún niño o padre rechazó el tratamiento con morfina para el dolor por miedo a la adicción [29].

En cuanto a la población general, dos estudios reportan la existencia de preocupación sobre el riesgo de adicción a opioides en el tratamiento del dolor en cáncer [66,67].

\section{Políticas, programas y protocolos de centros sanitarios acerca del uso inadecuado de opioides}

Dos artículos hacen referencia a la política de los centros sanitarios respecto al uso inadecuado de opioides $(68,69)$. En el primero de ellos se concluye que la mayoría de los centros de cuidados paliativos incluidos en el estudio no perciben el abuso de sustancias ni el desvío como un problema, no tienen protocolos establecidos y no realizan formación al respecto [68]. El segundo trabajo pretende medir los resultados de implantar un programa educacional dirigido a mejorar el uso y el almacenamiento de los opioides en pacientes oncológicos. De forma francamente interesante, dicha implantación consigue mejorar las conductas aberrantes disminuyendo el uso y almacenamiento inseguro de opioides en estos pacientes (69).

\section{DISCUSIÓN}

El objetivo de la presente revisión es actualizar la evidencia disponible en relación con el uso inadecuado de opioides en pacientes con dolor oncológico. Los estudios revisados son francamente heterogéneos en metodología y resultados, pero pueden agruparse entorno cuatro grandes áreas temáticas: epidemiología, factores de riesgo, opinión de los pacientes y los profesionales y políticas específicas. A continuación realizaremos un análisis y discusión breve de los principales resultados que hemos encontrado en cada una de ellas.

Respecto a la prevalencia de uso inadecuado de opioides o conductas aberrantes, los resultados obtenidos son muy heterogéneos tanto en población adulta como adolescente, describiéndose frecuencias que abarcan desde el 0 al $26 \%$ de uso inadecuado (32-37) y del 12 al $85 \%$ de conductas aberrantes (36-39). En nuestra opinión, las cifras de prevalencia baja, entorno al $0 \%$, podría estar influida por una baja detección del uso inadecuado de opioides, hecho que, de acuerdo con los resultados de Kwon y cols., podría relacionarse con el grado de especialización y conocimiento del personal sanitario responsable del paciente [35]. La baja detección de problemas relacionados con sustancias en pacientes con patología médica no es un tema nuevo y en las últimas décadas existen multiplicidad de trabajos al respecto. A modo de ejemplo podríamos citar el estudio ALCHIMIE que sitúa el porcentaje de detección del consumo perjudicial de alcohol en pacientes ingresados en Unidades de Medicina Interna de 43 hospitales europeos en tan solo el $50 \%$ (70).

Los resultados reportados en cuanto a los factores de riesgo son más homogéneos, describiéndose una mayor probabilidad de presentar un uso inadecuado de opioides en los pacientes de edad joven, con antecedentes psiquiátricos o de consumo de sustancias, con una intensidad elevada del dolor y con uso de dosis elevadas de opioides y/o únicamente de medicación de rescate $[35,39,47,49,50,51,53,56]$. Estas características descritas nos permiten establecer perfiles de pacientes con un riesgo mayor de presentar un uso inadecuado de opioides, paso fundamental para diseñar protocolos de intervención específicos encaminados a la prevención y la detección precoz de posibles conductas aberrantes. Algunos autores han intentado cuantificar el riesgo mediante diversos instrumentos entre los que destacarían por su frecuencia de uso el CAGE, el ORT o el SOAPP. Dichos trabajos ofrecen una amplia variabilidad de resultados, determinándose entre un 8 y un $43 \%$ de los pacientes con riesgo moderado-alto en función del instrumento utilizado $(50-52,56)$. Debido 
a esta disparidad de resultados sería probablemente aconsejable llegar a un consenso internacional para determinar la mejor metodología de detección de riesgo y de esta manera poder realizar políticas globales de detección e intervención.

En relación con la opinión de los profesionales, la impresión general es que actualmente el personal sanitario está más sensibilizado frente a esta problemática. En los primeros estudios realizados, los resultados pueden parecer contradictorios puesto que a pesar de la percepción de pocos casos de dependencia a opioides por parte de los profesionales, estos mostraban mayor dificultad en prescribir opioides $[30,57)$. Los estudios más recientes revelan una mayor inquietud sobre el uso inadecuado de opioides en pacientes oncológicos por parte de los profesionales $[35,58)$ y se ha llegado a un consenso internacional para la definición de afrontamiento químico del uso de opioides como el uso de los opioides para afrontar el malestar emocional que se caracteriza por un uso inadecuado y/o excesivo de opioide [53].

Referente a la percepción de los pacientes, es importante recalcar que la preocupación en relación con la adicción a opioides presente en la mitad de los pacientes oncológicos $[59,60)$ y la asociación del uso de morfina con el final de vida (62) interfiere notablemente en el manejo del dolor. Además, también son notorias las diferencias de resultados según el país donde se ha realizado el estudio.

En cuanto la existencia de políticas específicas en los centros sanitarios, y a pesar de la impresión de una mayor sensibilización al respecto, llama la atención la ausencia de protocolos y de formación especializada sobre esta problemática en centros de cuidados paliativos [68]. De manera interesante, De la Cruz y cols. [69] muestran una notable disminución en el uso y almacenamiento inseguro de opioides en pacientes oncológicos tras la implantación de un programa educacional para los pacientes. Por todo ello consideramos de gran importancia que se puedan diseñar e implementar programas de intervención específica destinados a la formación de los profesionales y la educación sanitaria de los pacientes.

\section{CONCLUSIONES}

Dados los resultados de la presente revisión, consideramos que es importante realizar un seguimiento pormenorizado y estructurado de cualquier paciente oncológico al que se inicia tratamiento opioide y, de manera más estricta, en aquellos perfiles descritos como de mayor riesgo. Dicho control longitudinal iría en la línea de la transición en el modelo de atención paliativa que se ha producido en las últimas décadas y que aboga por una atención paliativa precoz $(71,72)$. En este sentido consideramos que es fundamental establecer protocolos y políticas de detección precoz e intervención frente al uso inadecuado de opioides.

\section{FORTALEZAS Y LIMITACIONES}

Una potencial limitación de esta revisión es la heterogeneidad de los estudios incluidos en términos de objetivos, metodología y calidad. De todas maneras, la metodología de revisión sistemática integradora ha mostrado minimizar el riesgo de pasar por alto información relevante (73).

La principal fortaleza de esta revisión integradora es la búsqueda exhaustiva e inclusión de una selección de las publicaciones relevantes sobre el tema. Adicionalmente, esta metodología nos ha permitido sintetizar información de una amplia gama de fuentes, creemos que esto contribuye a una mejor comprensión al estudiar fenómenos desde una amplia gama de puntos de vista. A pesar de que el número de publicaciones acerca del uso inadecuado de opioides en pacientes oncológicos no es muy elevado, la presente revisión actualiza el conocimiento actual y proporciona tanto a clínicos como a investigadores una valiosa referencia para continuar trabajando en mejorar la atención de los pacientes. En este sentido los resultados de la revisión sistemática pueden representar un punto de partida sólido para diseñar protocolos de detección precoz e intervención en esta población y para realizar nuevos trabajos de investigación que aporten una mayor evidencia acerca de perfiles de pacientes de riesgo y estrategias de intervención exitosas.

\section{CONFLICTO DE INTERESES}

Los autores declaran no tener ningún conflicto de intereses.

\section{BIBLIOGRAFÍA}

1. Manual Diagnóstico y Estadístico de los Trastornos Mentales. $5^{a}$ ed. Arlington, VA: Asociación Americana de Psiquiatría; 2014.

2. Portenoy RK, Payne R. Acute and chronic pain. In: Lowinson $\mathrm{JH}$, Ruiz P, Millman RB (eds). Comprehensive Textbook of Substance Abuse. 3rd Edit. Baltimore: Williams and Wilkins; 1997. p. 564.

3. Webster L, St Marie B, McCarberg B, Passik SD, Panchal SJ, Voth E. Current status and evolving role of abuse-deterrent opioids in managing patients with chronic pain. J Opioid Manag. 2011;7(3):235-45. DOl: 10.5055/jom.2011.0066.

4. Passik SD, Weinreb HJ. Managing chronic nonmalignant pain: overcoming obstacles to the use of opioids. Adv Ther. 2000;17(2):70-83. DOI: 10.1007/BFO2854840.

5. Del Fabbro E. Assessment and management of chemical coping in patients with cancer. J Clin Oncol. 2014;32(16):1734-8. DOI: 10.1200/JC0.2013.52.5170.

6. Brown RL, Rounds LA. Conjoint screening questionnaires for alcohol and other drug abuse: criterion validity in a primary care practice. Wis Med J. 1995;94(3):135-40.

7. Webster LR, Webster RM. Predicting aberrant behaviors in opioid-treated patients: preliminary validation of the Opioid Risk Tool. Pain Med. 2005;6(6):434-42. DOI: 10.1111/j.1526-4637.2005.00072.x.

8. Akbik H, Butler SF, Budman SH, Fernandez K, Katz NP, Jamison RN. Validation and clinical application of the Screener and Opioid Assessment for Patients with Pain (SOAPP). J Pain Symptom Manage. 2006;32(3):287-93. DOI: 10.1016/j. jpainsymman.2006.03.010.

9. Butler SF, Budman SH, Fernandez KC, Houle B, Benoit C, Katz N, et al. Development and validation of the Current Opioide Misuse Measure. Pain. 2007;130(1-2):144-56. DOl: 10.1016/j.pain.2007.01.014.

10. Manchikanti L, Singh A. Therapeutic opioids: a ten-year perspective on the complexities and complications of the 
escalating use, abuse, and nonmedical use of opioids. Pain Physician. 2008; 11(2 Suppl):S63-88.

11. Jay GW, Barkin RL. Perspectives on the opioid crisis from pain medicine clinicians. Dis Mon. 2018;64(10):451-66. DOI: 10.1016/j.disamonth.2018.07.002.

12. Shipton EA, Shipton EE, Shipton AJ. A Review of the opioid epidemic: What do we do about it? Pain Ther. 2018;7(1):2336. DOI: 10.1007/s40122-018-0096-7.

13. U.S. Department of Justice - Drug enforcement administration - Diversion control division [Internet]. Springfield, VA. Disponible en: http://www.deadiversion.usdoj.gov/arcos/ retail_drug_summary/index.html.

14. Manchikanti L, Helm S 2nd, Fellows B, Janata JW, Pampati V, Grider JS, et al. Opioid epidemic in the United States. Pain Physician. 2012; 15(3 Suppl):ES9-38.

15. Madadi P, Hildebrandt D, Lauwers AE, Koren G. Characteristics of opioid-users whose death was related to opioid-toxicity: a population-based study in Ontario, Canada. PLoS One. 2013;8(4):e60600. DOl: 10.1371/journal.pone.0060600.

16. Schubert I, Ihle P, Sabatowski R. Increase in opiate prescription in Germany between 2000 and 2010: a study based on insurance data. Dtsch Arztebl Int. 2013;110(4):45-51. DOI: 10.3238/arztebl.2013.0045.

17. Carmichael AN, Morgan L, Del Fabbro E. Identifying and assessing the risk of opioid abuse in patients with cancer: an integrative review. Subst Abuse Rehabil. 2016;7:71-9.

18. Sociedad científica española de estudios sobre el alcohol, el alcoholismo y las otras toxicomanías (Socidrogalcohol). Guía de consenso para el buen uso de analgésicos opioides en el dolor crónico. 1 ${ }^{\text {a }}$ ed, 2017. ISBN: 978-84-945737-4-3.

19. Miaskowski C, Dodd MJ, West C, Paul SM, Tripathy D, Koo $P$, et al. Lack of adherence with the analgesic regimen: a significant barrier to effective cancer pain management. J Clin Oncol. 2001;19(23):4275-9. DOI: 10.1200/ JCO.2001.19.23.4275.

20. Nguyen LM, Rhondali W, De la Cruz M, Hui D, Palmer L, Kang $\mathrm{DH}$, et al. Frequency and predictors of patient deviation from prescribed opioids and barriers to opioid pain management in patients with advanced cancer. J Pain Symptom Manage. 2013;45(3):506-16. DOI: 10.1016/j.jpainsymman.2012.02.023.

21. Mayahara M, Foreman MD, Wilbur J, Paice JA, Fogg LF. Effect of hospice nonprofessional caregiver barriers to pain management on adherence to analgesic administration recommendations and patient outcomes. Pain Manag Nurs. 2015;16(3):249-56. DOI: 10.1016/j.pmn.2014.07.001.

22. Hojsted J, Sjogren P. Addiction to opioids in chronic pain patients: a literature review. Eur J Pain. 2007;11(5):490518. DOI: 10.1016/j.ejpain.2006.08.004.

23. Minozzi S, Amato L, Davoli M. Development of dependence following treatment with opioid analgesics for pain relief: a systematic review. Addiction. 2013;108(4):688-98. DOI: 10.1111/j.1360-0443.2012.04005.x.

24. Moher D, Liberati A, Tetzlaff J, Altman DG; PRISMA Group. Preferred reporting items for systematic reviews and meta-analyses: the PRISMA statement. J Clin Epidemiol. 2009;62(10):100612. DOI: 10.1016/j.jclinepi.2009.06.005.

25. Whittemore R, Knafl K. The integrative review: updated methodology. J Adv Nurs. 2005;52(5):546-53. DOI: 10.1111/j.1365-2648.2005.03621.x.

26. Carroll C, Booth A, Lloyd-Jones M. Should we exclude inadequately reported studies from qualitative systematic reviews? An evaluation of sensitivity analyses in two case study reviews. Qual Health Res. 2012;22(10):1425-34. DOI: $10.1177 / 1049732312452937$.
27. Kanner RM, Foley KM. Patterns of narcotic drug use in a cancer pain clinic. Ann N Y Acad Sci. 1981;362:161-72. DOI: 10.1111/j.1749-6632.1981.tb12804.x.

28. Sun WZ, Chen TL, Fan SZ, Peng WL, Wang MS, Huang FY. Can cancer pain attenuate the physical dependence on chronic long-term morphine treatment? J Formos Med Assoc. 1992;91(5):513-20.

29. Ljungman G, Gordh T, Sörensen S, Kreuger A. Pain in paediatric oncology: interviews with children, adolescents and their parents. Acta Paediatr. 1999;88(6):623-30. DOI: 10.1080/08035259950169279.

30. Vainio A. Practising physicians' experiences of treating patients with cancer pain. Acta Oncol. 1989;28(2):177-82. DOI: 10.3109/02841868909111243.

31. Tabei SZ, Heydari ST, Mehrabani D, Shamsina SJ, Ahmadi J, Firouzi SM. Current substance use in patients with gastric cancer in Southern Iran. J Cancer Res Ther. 2006;2(4):1825. DOI: 10.4103/0973-1482.29828.

32. Santana Pineda MM, Jover López-Rodrigálvarez R, Rodríguez Sainz P, Gómez Cortes MD, Rodríguez Huertas F, Morgado Muñoz I. Uso y abuso de opioides en el área norte de la provincia de Cádiz. Rev Soc Esp Dolor. 2016;23(3):127-34.

33. Payne R, Coluzzi P, Hart L, Simmonds M, Lyss A, Rauck $R$, et al. Long-term safety of oral transmucosal fentanyl citrate for breakthrough cancer pain. J Pain Symptom Manage. 2001;22(1):575-83. DOI: 10.1016/s08853924(01)00306-2.

34. Taylor D, Radbruch L, Revnic J, Torres LM, Ellershaw JE, Perelman M. A report on the long-term use of fentanyl pectin nasal spray in patients with recurrent breakthrough pain. J Pain Symptom Manage. 2014;47(6):1001-7. DOI: 10.1016/j.jpainsymman.2013.07.012.

35. Kwon JH, Tanco K, Park JC, Wong A, Seo L, Liu D, et al. Frequency, predictors, and medical record documentation of chemical coping among advanced cancer patients. Oncologist. 2015;20(6):692-7. DOl: 10.1634/theoncologist.2015-0012.

36. Reddy A, de la Cruz M, Rodriguez EM, Thames J, Wu J, Chisholm G, et al. Patterns of storage, use, and disposal of opioids among cancer outpatients. Oncologist. 2014;19(7):7805. DOI: 10.1634/theoncologist.2014-0071.

37. Silvestre J, Reddy A, de la Cruz M, Wu J, Liu D, Bruera E, et al. Frequency of unsafe storage, use, and disposal practices of opioids among cancer patients presenting to the emergency department. Palliat Support Care. 2017;15(6):638-43. DOI: $10.1017 /$ S1478951516000158.

38. Anghelescu DL, Ehrentraut JH, Faughnan LG. Opioid misuse and abuse: risk assessment and management in patients with cancer pain. J Natl Compr Canc Netw. 2013;11(8):102331. DOl: 10.6004/jnccn.2013.0120.

39. Ehrentraut $\mathrm{JH}$, Kern KD, Long $S A, A n A Q$, Faughnan LG, Anghelescu DL. Opioid misuse behaviors in adolescents and young adults in a hematology/oncology setting. J Pediatr Psychol. 2014;39(10):1149-60. DOl: 10.1093/jpepsy/jsu072.

40. Evans PJ. Narcotic addiction in patients with chronic pain. Anaesthesia 1981;36(6):597-602. DOI: 10.1111/j.13652044.1981.tb10323.x.

41. Macaluso C, Weinberg D, Foley KM. Opioid abuse and misuse in a cancer pain population. J Pain Symptom Manage 1988;3:S24.

42. Schug SA, Zech D, Grond S, Jung H, Meuser T, Stobbe $B$. A long-term survey of morphine in cancer pain patients. J Pain Symptom Manage. 1992;7(5):259-66. DOI: 10.1016/0885-3924(92)90059-q.

43. Passik SD, Kirsh KL, McDonald MV, Ahn S, Russak SM, Martin L, et al. A pilot survey of aberrant drug-taking attitu- 
des and behaviors in samples of cancer and AIDS patients. J Pain Symptom Manage. 2000;19:274-86. DOI: 10.1016/ s0885-3924(00)00119-6.

44. Passik SD, Schreiber J, Kirsh KL. A chart review of the ordering of urine toxicology screen in a cancer center: do they influence on pain management. J Pain Symptom Manage. 2000;19(1):40-4. DOl: 10.1016/s0885-3924(99)00137-2.

45. Passik SD, Kirsh KL, Donaghy KB, Portenoy RK. Pain and aberrant drug-related behaviors in medically ill patients with and without histories of substance abuse. Clin J Pain. 2006;22(2):173-81. DOI: 10.1097/01. ajp.0000161525.48245.aa.

46. Hojsted J, Nielsen PR, Guldstrand SK, Frich L, Sjogren P. Classification and identification of opioid addiction in chronic pain patients. Eur J Pain. 2010;14(10):1014-20. DOI: 10.1016/j.ejpain.2010.04.006.

47. Bohnert AS, Valenstein M, Bair MJ, Ganoczy D, McCarthy JF, Ilgen MA, et al. Association between opioid prescribing patterns and opioid overdose-related deaths. JAMA. 2011;305(13):1315-21. DOI: 10.1001/jama.2011.370.

48. Novy DM, Lam C, Gritz ER, Hernandez M, Driver LC, Koyyalagunta $\mathrm{D}$. Distinguishing features of cancer patients who smoke: pain, symptom burden, and risk for opioid misuse. J Pain. 2012;13(11):1058-67. DOl: 10.1016/j.jpain.2012.07.012.

49. Koyyalagunta D, Bruera E, Aigner C, Nusrat H, Driver L, Novy D. Risk stratification of opioid misuse among patients with cancer pain using the SOAPP-SF. Pain Med. 2013;14(5):667-75. DOI: 10.1111/pme.12100.

50. Barclay JS, Owens JE, Blackhall LJ. Screening for substance abuse risk in cancer patients using the Opioid Risk Tool and urine drug screen. Support Care Cancer. 2014;22(7):18838. DOI: 10.1007/s00520-014-2167-6.

51. Ma JD, Horton JM, Hwang M, Atayee RS, Roeland EJ. A single-center, retrospective analysis evaluating the utilization of the opioid risk tool in opioid-treated cancer patients. J Pain Palliat Care Pharmacother. 2014;28(1):4-9. DOI: 10.3109/15360288.s2013.869647.

52. Childers JW, King LA, Arnold RM. Chronic pain and risk factors for opioid misuse in a palliative care clinic. Am J Hosp Palliat Care. 2015;32(6):654-9. DOI: 10.1177/1049909114531445.

53. Kwon JH, Hui D, Bruera E. A pilot study to define chemical coping in cancer patients using the Delphi method. J Palliat Med. 2015;18(8):703-6. DOI: 10.1089/jpm.2014.0446.

54. Arthur JA, Edwards T, Lu Z, Reddy S, Hui D, Wu J, et al. Frequency, predictors, and outcomes or urine drug testing among patients with advanced cancer on chronic opioid therapy at an outpatient supportive care clinic. Cancer. 2016;122(23):3732-9. DOI: 10.1002/cncr.30240.

55. Kim YJ, Dev R, Reddy A, Hui D, Tanco K, Park M, et al. Association between tobacco use, symptom expression, and alcohol and illicit drug use in advanced cancer patients. J Pain Symptom Manage. 2016;51(4):762-8. DOI: 10.1016/j. jpainsymman.2015.11.012.

56. Reyes-Gibby CC, Anderson KO, Todd KH. Risk for opioid misuse among emergency department cancer patients. Acad Emerg Med. 2016;23(2):151-8. DOI: 10.1111/ acem. 12861.

57. Von Roenn JH, Cleeland CS, Gonin R, Hatfield AK, Pandya KJ. Physician attitudes and practice in cancer pain management. A survey from the Eastern Cooperative Oncology Group. Ann Intern Med. 1993;119(2):121-6. DOI: 10.7326/00034819-119-2-199307150-00005.

58. Serra E, Marchand F, Mimassi N, Ganry H. Point de vue des médecins généralistes sur les risques de survenue d'une dépendance lors de la prescription d'opioïdes forts. Ann Med Psychol (Paris). 2014;172(4):323-9.

59. Paice JA, Toy C, Shott S. Barriers to cancer pain relief: fear of tolerance and addiction. J Pain Symptom Manage. 1998;16(1):1-9. DOI: 10.1016/s0885-3924(98)00025-6.

60. Wills BS, Wootton YS. Concerns and misconceptions about pain among Hong Kong Chinese patients with cancer. Cancer Nurs. 1999;22(6):408-13. DOl: 10.1097/00002820$199912000-00002$.

61. Davies AN, Vriens J, Kennett A, McTaggart M. An observational study of oncology patients' utilization of breakthrough pain medication. J Pain Symptom Manage. 2008;35(4):40611. DOI: 10.1016/j.jpainsymman.2007.05.010.

62. Reid CM, Gooberman-Hill R, Hanks GW. Opioid analgesics for cancer pain: symptom control for the living or comfort for the dying? A qualitative study to investigate the factors influencing the decision to accept morphine for pain caused by cancer. Ann Oncol. 2008;19(1):44-8. DOI: 10.1093/ annonc/mdm462.

63. Jacobsen R, Samsanaviciene J, Liuabarskiene Z, Sciupokas $A$. Barriers to pain management among Lithuanian cancer patients. Pain Pract. 2010;10(2):145-57. DOI: 10.1111/j.1533-2500.2009.00333.x.

64. Alsirafy SA, Saleh RN, Fawzy R, Alnagar AA, Hammad AM, El-Sherief $W$, et al. The fear of using tramadol for pain control (tramadolophobia) among Egyptian patients with cancer. J Opioid Manag. 2015;11(6):474-80. DOI: 10.5055/ jom.2015.0300.

65. Cella IF, Trindade LCT, Sanvido LV, Skare TL. Prevalence of opiophobia in cancer pain treatment. Rev Dor. Sao Paulo. 2016;17(4):245-7.

66. Levin DN, Cleeland CS, Dar R. Public attitudes toward cancer pain. Cancer. 1985;56(9):2337-9. DOI: 10.1002/1097-0142 (19851101)56:9<2337::aid-cncr2820560935>3.0.co;2-w.

67. Grant M, Ugalde A, Vafiadis P, Philip J. Exploring the myths of morphine in cancer: views of the general practice population. Support Care Cancer. 2015;23(2):438-9. DOI: 10.1007/ s00520-014-2379-9.

68. Blackhall LJ, Alfons ED, Barclay JS. Screening for substance abuse and diversion in Virginia hospices. J Palliat Med. 2013;16(3):237-42. DOI: 10.1089/jpm.2012.0263.

69. De la Cruz M, Reddy A, Balankari V, Epner M, Frisbee-Hume S, $\mathrm{Wu}$ J, et al. The impact of an educational program on patient practices for safe use, storage, and disposal of opioids at a comprehensive cancer center. Oncologist. 2017;22(1):11521. DOI: 10.1634/theoncologist.2016-0266.

70. Rosón B, Corbella X, Perney P, Santos A, Stauber R, Lember $M$, et al. Prevalence, Clinical Characteristics, and Risk Factors for Non-recording of Alcohol Use in Hospitals across Europe: The ALCHIMIE Study. Alcohol Alcohol. 2016;51(4):457-64. DOI: 10.1093/alcalc/agv142.

71. Ferrel BR, Temel JS, Temin S, Alesi ER, Balboni TA, Basch EM, et al. Integration of palliative care into standard oncology care: american society of clinical oncology clinical practice guideline update. J Clin Oncol. 2017;35(1):96-112.

72. Kaasa S, Loge JH, Aapro M, Albreht T, Anderson R, Bruera E, et al. Integration of oncology and palliative care: a Lancet Oncology Comission. Lancet Oncol. 2018;19(11):e588-e653. DOI: 10.1016/S1470-2045(18)30415-7.

73. Higginson IJ, Evans CJ, Grande G, Preston N, Morgan M, McCrone $P$, et al. Evaluating complex interventions in end of life care: the MORECare statement on good practice generated by a synthesis of transparent expert consultations and systematic reviews. BMC Med. 2013;11:111. DOI: 10.1186/1741-7015-11-111. 